\title{
Diapycnal Mixing and Internal Waves
}

\author{
Peter Miiller \\ University of Hazuai - Honolulu, Haznaii USA \\ Melloume Briscoe \\ Office of Naval Research • Arlington, Virginia USA
}

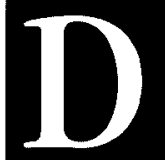

iapycnal mixing is an integral factor in the meridional overturning circulation of the ocean. The slow upwelling in the ocean interior across density surfaces requires counteracting diapycnal diffusion. Diapycnal diffusion in a stably stratified fluid requires mechanical energy and the availability of this energy might well be the controlling factor in setting the strength of the overturning circulation as, e.g., discussed by Munk and Wunsch $(1997,1998)$. The conventional wisdom is that

- Diapycnal mixing in the ocean interior is driven by intermittent patches of small scale turbulence.

- The turbulent patches have a vertical extent of up to a few meters and are caused by breaking internal gravity waves.

- Internal waves break by either shear or convective instabilities that are caused by chance superpositions or encounters with critical layers.

Confirming this connection between oceanic internal gravity waves and diapycnal mixing motivates and leads to funding for a large part of internal wave research. By understanding the internal wave field we may understand diapycnal mixing. Diapycnal mixing can also be caused by double diffusion, but this process will not be considered here.

What is the state of our understanding of the oceanic internal wave field? This was the task put before the participants of the eleventh 'Aha Huliko'a Hawaiian Winter Workshop, held from January 18 to 22, 1999, in Honolulu, Hawaii. The meeting came at an opportune time. Major field programs had just been completed (e.g., the Littoral Internal Wave Initiative [LIWI], the Brazil Basin Experiment, the Internal Tide Experiments [ITEX], the Internal Waves on the Continental Margin [IWAVES] experiment). The interaction of internal waves with topography had extensively been studied and the significance of internal wave induced boundary mixing had been explored. It was hoped that formerly incongruent pieces could be synthesized into a coherent and consistent picture of internal wave dynamics and internal wave induced mixing. However, the pieces still do not fit together. There was a feeling at the workshop that perhaps a break with basic underlying assumptions, a change of paradigm, is necessary. Below is the more detailed story.

\section{The Garrett and Munk Spectrum}

Much of internal wave research has been organized around the Garrett and Munk spectrum. More than twenty five years ago Garrett and Munk (1972) synthesized existing observations into a kinematic model spectrum that describes the observed distribution of internal wave energy in wavenumber and frequency space. Most of the detailed features of this spectrum were confirmed by the tri-moored internal wave experiment IWEX (Müller et al., 1978). The GM spectrum, as it is known now, describes a wave field that is horizontally isotropic (waves coming in from all horizontal directions equally) and vertically symmetric (as many waves are propagating downward as upward). Often upward and downward propagating waves are assumed to be phaselocked and to produce vertically standing "normal modes." The most significant and surprising aspect of the GM spectrum is its universality. To observe significant (say larger than a factor of three) deviations from the GM spectrum one has to go to very special places such as the Arctic Ocean or submarine canyons. Figure 1 shows a typical example of how well the GM model represents an observed frequency spectrum.

It should be stressed that the GM spectrum does not describe all of the variance of the internal wave field. It only describes the "continuum," the broad background with a minus two slope in Figure 1, and the inertial peak. It does not account for the baroclinic (or internal) tidal peaks, which contain a considerable but highly variable fraction of the variance. In addition, the GM spectrum does not properly represent many of the observed properties of the inertial peak. It neither reflects its large temporal variability, nor its strong dependence on depth, nor its vertical asymmetry (most near-inertial waves are observed to propagate downwards). 
Since the formulation of the GM spectrum the focus has shifted from measuring the energy of the wave field, which is determined by the velocity and displacement variance, to measuring dynamically more active quantities, such as the shear or rate of strain. As observational techniques were developed to explore the wavenumber-frequency regions where the variance of these quantities is concentrated, it was found that other aspects of the GM spectrum also did not hold up, such as the separability of wavenumber and frequency dependence. We will not dwell on these aspects here.

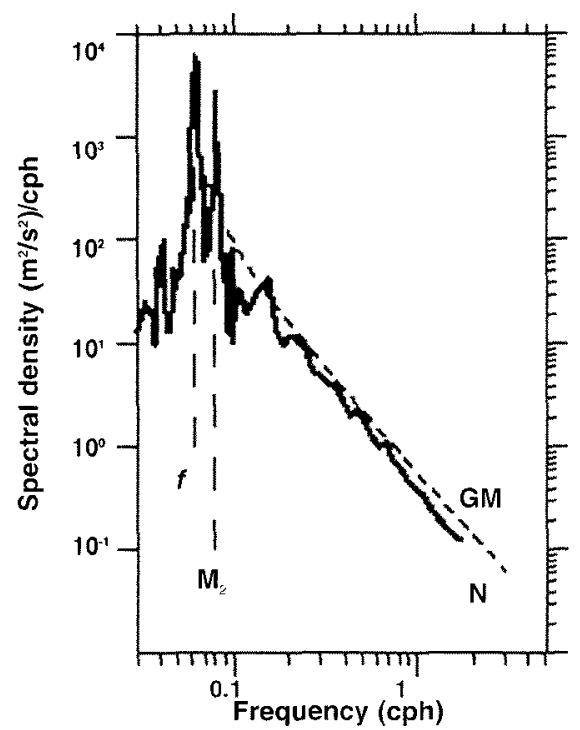

Figure 1: Fremuency spectrum of the horizontal kinetic chergy at $140 \mathrm{~m}$ depth at a site in the northeast Pacific during the Ocean Stoms Expcrintent, 1987-88. Internal waves exist betwecn the Coriolis frequentay and the buoyancy frequency N. $M_{2}$ is the semi-diumal tidal friquency. The dashed line labeled GM is the Garrett and Munk model prediction. (Coutesy of M. Levine.)

\section{The Dynamic Balance}

Given a kinematic description of the internal wave field, the community started to search for a dynamical explanation of the spectrum as a balance of generation, transfer and dissipation processes. There are many dynamical processes that affect internal waves. A suitable theoretical framework for their quantitative calculation is the radiation balance equation, which describes the evolution of the wave spectrum along wave group trajectories. The dynamic balance that came out of these efforts is depicted in Figure 2.

This conventional dynamic balance has only two energy sources for internal waves: (i) changes in the atmospheric windstress cause inertial oscillations in the oceanic surface mixed layer; part of their energy propagates into the ocean interior as large-scale, near-inertial internal waves; (ii) barotropic (or surface) tidal currents impinging on topography in a stratified ocean generate internal waves of tidal frequency, the baroclinic tides.
Their large-scale components propagate into the ocean interior. There is good observational evidence for both these generation processes. Near-inertial internal waves have been observed to propagate away from the surface underneath storm and cyclone tracks. Baroclinic tidal beams have been observed to emanate from certain topographic features and can often be traced for hundreds of kilometers. As these large-scale waves propagate away from their generation region, they are assumed to interact nonlinearly with themselves and other internal waves and to scatter at mesoscale eddies, fronts, and topography. These nonlinear interaction and scattering processes transfer energy out of the largescale waves into ever smaller scale waves that eventually break and cause turbulence and mixing. In a breaking event, the wave energy is partly dissipated into heat and partly converted to potential energy, since the mixing of a stably stratified fluid increases its potential energy. The nonlinear cascade to small scales by wavewave interactions has been theoretically investigated in the resonant and eikonal approximations. Attempts to observationally confirm this nonlinear cascade by bispectral analysis have failed because the instationarity and inhomogeneity of the ocean environment masked the expected signal.

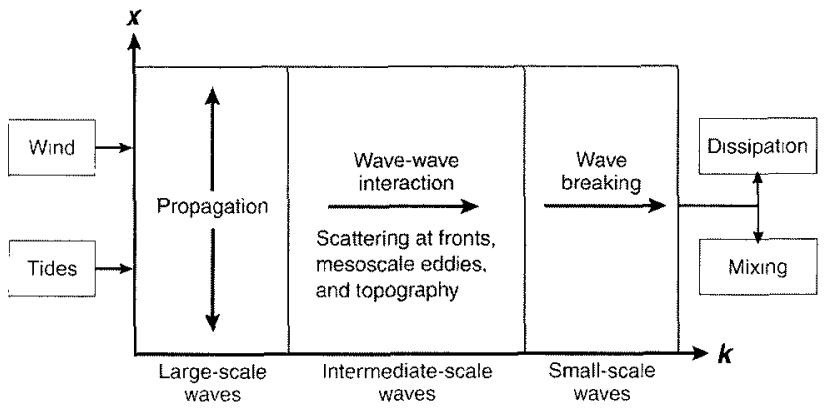

Figure 2: The conentional dynamic balance of the oceanic internal wave field in physical $(x)$ and ardenumber $(k)$ spacc. The wind and tides generate large-scale wates of nour inertial and tidal frequemcies. These large-sonle araes propagate aicay from their sources in physical space and cascade towards small-scale wates in wave-number space. The cascade is antsed by ware-ane interactions and scattering at fronts, mesoscale eddics, topography, and other scatterers. The sntall-scale wawes break and cause turblence and mixing.

The major confirmation of the scenario shown in Figure 2 comes from microstructure measurements. Over the last two decades microstructure measurements have been collected in a wide variety of ocean conditions, in conjunction with internal wave measurements. From microstructure measurements dissipation and mixing rates can be inferred. It was found that these dissipation rates scale with the internal wave energy and shear level as predicted by the nonlinear cascade calculation. This quantitative connection between 
microstructure measurements and internal wave calculations is what gave credibility to the scenario in Figure 2. It also revived the concept of boundary mixing, as we will discuss next.

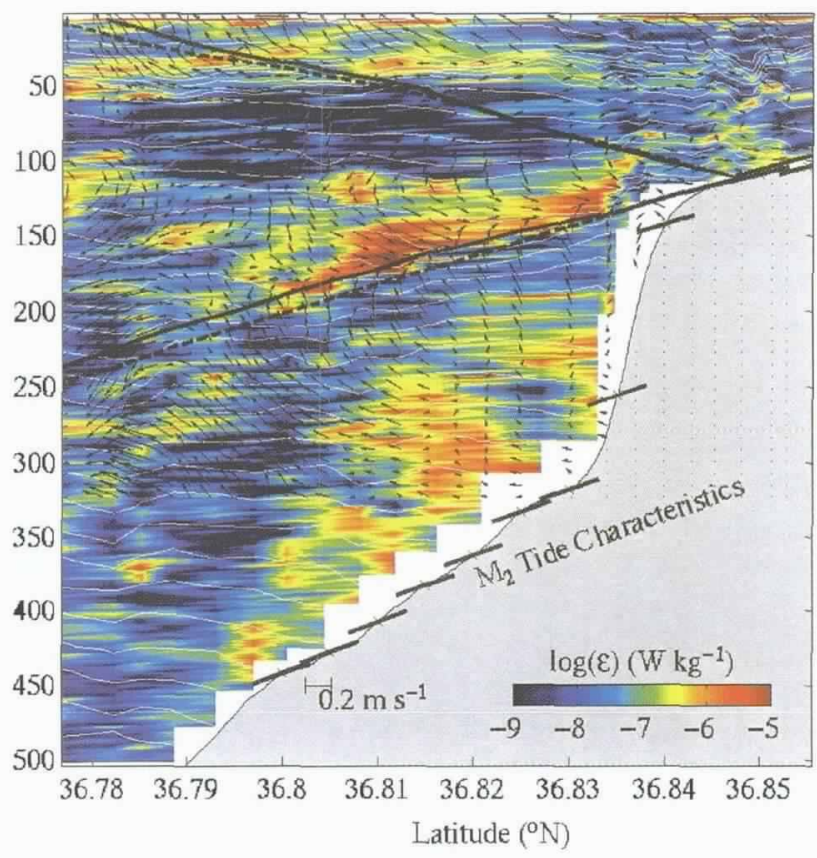

Figure 3. Section of the dissipation rate and velocity along the ridge of a sea fan outside Monterey Bay, California. Solid and dashed lines are characteristics of the internal tide, using different values of the bouyancy frequency. The dissipation rates are high along the internal tide characteristics and in a thick layer of strongly stratified water above the bottom, indicating zones of intense mixing (courtesy of $M$. Gregg).

\section{Boundary Mixing}

The measured and deduced dissipation or mixing rates are equivalent to a diapycnal diffusivity of $10^{-5} \mathrm{~m}^{2} \mathrm{~s}^{-1}$. This is one order of magnitude smaller than the value of $10^{-4} \mathrm{~m}^{2} \mathrm{~s}$, which has been inferred from the abyssal oceans' mass and heat balances and is required to maintain the abyssal stratification. Internal wave induced mixing in the ocean interior is not efficient enough to provide the mixing that is needed. The main mixing areas must lie elsewhere. The sloping side boundaries were immediately suspected as these mixing areas and this suspicion was nurtured by recent observations that show highly elevated dissipation levels near topography, as in Figure 3.

Internal waves can easily be implicated in causing this boundary mixing. Internal waves radiate a sufficiently large energy flux onto topography, about $20 \mathrm{~mW} \mathrm{~m}$ for the GM spectrum. It has been estimat- ed that if only $1 \mathrm{~mW} \mathrm{~m}^{-2}$ or $5 \%$ of this flux could be converted to mixing it would sustain a diapycnal diffusivity of $10^{-4} \mathrm{~m}^{2} \mathrm{~s}^{-1}$ in the ocean interior. This conversion might be facilitated by a peculiar property of internal waves. When reflecting from a sloping surface the wave vector of an internal wave maintains its angle with the vertical rather than with the normal vector of the reflecting surface, as in specular reflection. This reflection law implies "critical reflection" when this angle becomes equal to the angle of the normal vector. The reflected wave then has an infinite wavenumber and zero group velocity. Energy density and shear increase beyond bound at the reflecting surface. Wave breaking and mixing occur. Thus reflection or, more generally, scattering at topography is a way to make internal wave energy available for mixing. It was hoped that such scattering in conjunction with suitable processes that will restratify the mixed water and communicate the mixing to the interior would account for an effective diapycnal diffusivity of $10^{-4} \mathrm{~m}^{2} \mathrm{~s}^{-1}$. One such process is flow separation, a vigorous process that sweeps the mixed water into the interior and replenishes it with stratified water.

This is, in broad strokes, the current state of the relation between internal waves and diapycnal mixing and the state critically reviewed by the participants of the workshop. Of course, this review showed that the situation is not this simple.

\section{Interaction with Topography}

First, the interaction of internal waves with topography is an extremely complex problem. Even the reflec-

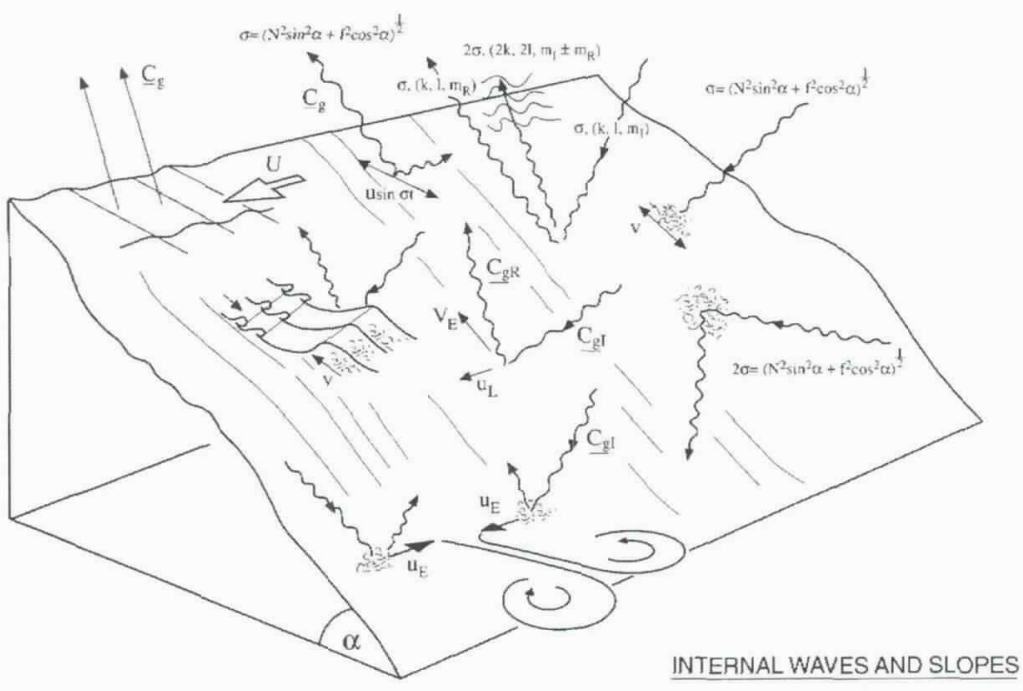

Figure 4: Physical processes involving the interaction of internal waves on sloping topography. Moving from the top left to right these are (i) lee wave generation by flow over topography, (ii) wave generation by oscillafory flow, particularly those of tidal period, (iii) resonant interaction between incident and reflected waves and, (iv) wave breaking when the incident is near or at critical frequency. The second row, left to right, shows (v) wave steepening and the formation of fronts upon reflection, (vi) the generation of upslope Eulerian mean flows, $V_{E}$, and along-slope Lagrangian mean flows, $U_{1}$, as waves reflect, and (vii) mixing produced by reflecting subcritical waves when the first harmonic is near critical. The final illustration, bottom left, represents Eulerian mean flows, $U_{E}$, generated by waves, which break and lose momentum at the boundary (courtesy of S. Thorpe). 
tion at an infinite sloping plane becomes somewhat involved when non-normal incidence is considered. Once finite topography in a finite-depth ocean is considered one must resort to numerical procedures, even for the linear problem. Results depend sensitively on the characteristics of the topography, such as the curvature and the amount and type of small-scale irregularities. Once nonlinear effects are included, a whole suite of new processes emerges, as depicted in Figure 4, involving secondary and tertiary flows, Lagrangian and Eulerian mean flows, and cross and along-slope flows. Again, non-normal incidence is the most challenging problem. Overall, it is fair to say that we have only started to identify and quantify all the different types of possible interactions. Given a topography, we cannot yet reliably estimate the amount of boundary mixing caused by an incident internal wave field. The interaction of internal waves with topography remains an active and important research area.

Second, the new research results presented at the workshop also questioned the conventional dynamic balance shown in Figure 2. It needs to be replaced by the extended dynamic balance shown in Figure 5, which clearly distinguishes between the baroclinic tides, the inertial peak and the continuum.

\section{The Baroclinic Tides}

When the barotropic tides impinge on continental slopes, mid-ocean ridges, sills or other topographic features, they generate a variety of baroclinic wave motions. These baroclinic wave motions contain the fundamental tidal frequency and all of its harmonics. The baroclinic waves propagate away from the topography or are trapped to it. The trapped waves dissipate their energy locally at the topography and contribute to boundary mixing. The propagating waves become part of the radiative internal wave field in the ocean interior. These energy pathways are depicted in Figure 5. The baroclinic tidal box is denoted by " $\mathrm{M}_{3}$ " the major tidal constituent. The details of the conversion of barotropic to baroclinic tidal energy, the partition into propagating and trapped waves, and the associated mixing depend on the tidal frequency and on the details of the topography and environment and are not fully understood yet. The conversion is particularly efficient at critical slopes where the bottom slope matches the slope of the internal tide characteristics (see Figure 3). The study and quantification of these conversion and mixing processes is the goal of the Hawaiian Ocean Mixing Experiment (HOME) that commenced in 1999.

Baroclinic tides propagating onto the shelf often steepen as they shoal and form bores, solitons, and other interesting nonlinear waveforms. The observations and theories of these nonlinear waveforms were the exclusive topic of the 1998 IOS/WHOI/ONR Internal Solitary Wave Workshop in Victoria, British Columbia, Canada, and were only briefly reviewed at the workshop.

\section{Near-Inertial Waves}

Near-inertial internal waves also deserve their own box in Figure 5, denoted by " $\mathrm{f}$ ". They are generated by the wind, most vigorously under storm tracks in winter and cyclone tracks in fall. Thereafter they propagate equatorward. A particular scenario suggests that these waves propagate equatorward until their frequency exceeds twice the local Coriolis frequency. Then the waves become susceptive to parametric sub-harmonic instability and subsequent wave breaking. Numerical modeling of these processes in the North Pacific shows that a very fragmented internal wave field emerges from this scenario, with internal-wave energy and mixing only occurring in certain parts of the Pacific Ocean in certain seasons. This energy pathway may, however, be obstructed. Near-inertial waves scatter off bottom topography many times before they get far enough equatorward to undergo instability.

Near-inertial waves are also observed in the vicinity of the equator, generated by westerly wind bursts. Though these waves have periods of weeks and wavelengths of thousands of kilometers, they are internal gravity waves. They obey the internal wave propagation laws just as the shorter period and smaller wavelength manifestations at midlatitudes.

\section{The Continuum}

The dynamic balance of the continuum is much less understood. The link that is most clear is the calculation of the nonlinear energy flux from low to high wavenumbers within the continuum. There are still some numerical differences between the different theories, but it was felt that these discrepancies will resolve themselves when a clearer account is taken of what interactions are included and excluded in the various approaches. Within the conventional balance, the energy of the continuum is assumed to come from the baroclinic tides and from inertial waves. However both these sources are highly localized. Near-inertial internal waves are mostly generated at the surface under storm and cyclone tracks. Baroclinic tides only emanate from certain topographic features. This strong localization of the sources does not immediately square with the universal, horizontally isotropic, and vertically symmetric GM spectrum.

The conventional way out of this dilemma is to argue that large-scale near-inertial waves and baroclinic tides propagate long distances, thereby obscuring their sources and providing more uniformly distributed sources for the continuum. However, these assertions have not been substantiated as yet. There have been no systematic studies of which parts of the ocean are "illuminated" by the assumed internal wave sources; nor have the mechanisms been identified by which energy is extracted from the large-scale propagating waves and transferred to the continuum. A rariant of this scenario is to assume that there exist scatterers in the ocean, also not identified, that turn an internal wave beam into an 


\section{Extended dynamic balance}

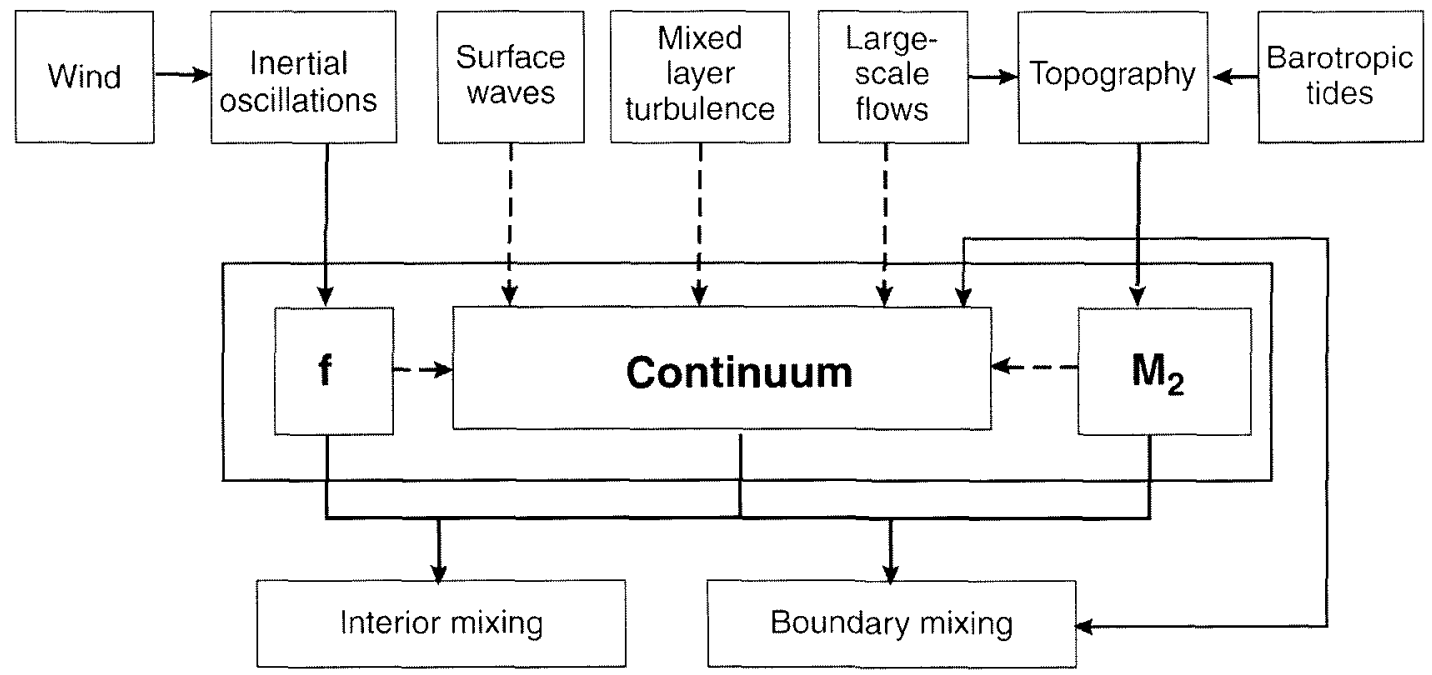

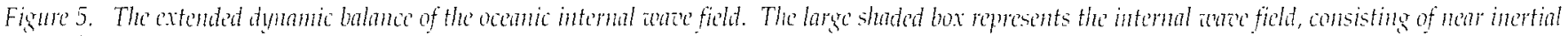

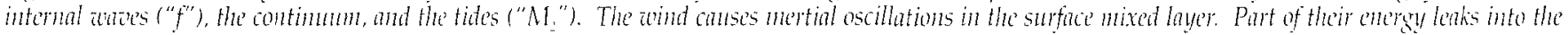

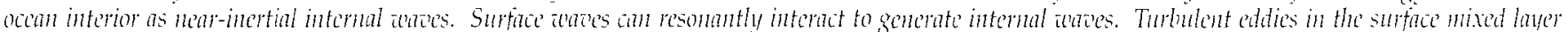

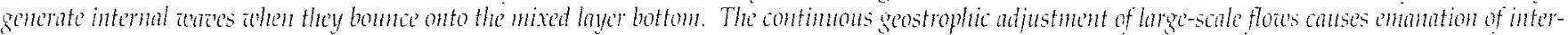

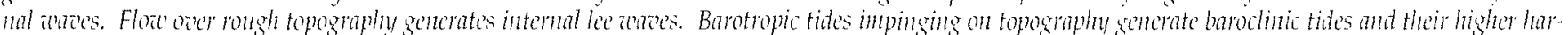

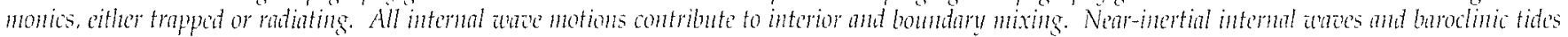

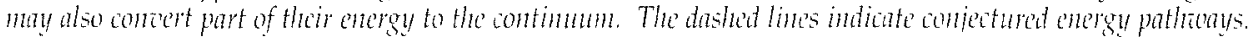

isotropic wave field, similar to the way water droplets in a cloud turn the incoming sun light into diffuse light.

Another way out of this dilemma is that there exist strong but yet overlooked sources for the continuum that are more widely distributed. Several such sources were discussed at the workshop:

- Generation of internal lee waves by flows over rough topography. There is observational evidence from the Brazil Basin Experiment that this mechanism is indeed a significant source of internal wave energy and that this energy is dissipated in the water column above.

- Generation of internal waves by the continuous geostrophic adjustment of large-scale flows.

- Generation by turbulent eddies in the mixed layer. As these eddies bounce onto the mixed layer bottom, they generate interfacial waves, which then propagate into the interior. Turbulence can thus be a source rather than a sink of internal wave energy.

- Generation by surface waves. Two swell components can interact resonantly to generate one internal wave. This is a somewhat forgotten but potentially efficient process, depending on the stratification and swell characteristics. The upper ocean internal wave energy level has been observed to vary with the swell intensity, however with an eleven day lag (Briscoe, 1983).

All these processes are shown in the extended energy diagram, Figure 5.

There was also a more radical faction at the workshop that argued that perhaps the universal isotropic GM spectrum is a myth, that the oceanic internal wave field consists instead of highly intermittent and directional wave groups. Perhaps the universality and isotropy are just a consequence of the way we analyze and present data. Stable spectral estimates require considerable averaging, thereby eliminating finegrained information about the wave field. Also, log-log representations of spectra, as in Figure 1, tend to emphasize power laws and the continuum. The exact kinematic structure of the internal wave field also has dynamical implications. The extent of "groupiness" affects the patterns and observable signatures of wave breaking.

In order to resolve the discrepancy between the stationary, homogeneous, and isotropic GM spectrum and the highly localized energy sources we need to continue to study environments where the internal wave spectrum deviates from the GM spectrum (Wunsch, 1976). Indeed, the workshop saw many new observations of internal waves and microstructure in canyons, fjords, and inlets and on the continental slope and shelf and theoretical and numerical efforts to understand the dynamics of internal waves in these peculiar environments.

\section{Summary and Outlook}

The eleventh 'Aha Huliko'a Workshop reviewed our understanding of the oceanic internal wave field and internal wave induced diapycnal mixing. It became clear at the workshop that a clear distinction needs to be 
made between the inertial peak, the baroclinic tidal peak, and the continuum. The energy in the inertial and tidal peaks is highly variable; the energy level of the continuum is "universal" and represents a stationary, homogeneous, isotropic wave field that is well described by the Garrett and Munk kinematic model spectrum, subject to minor modifications. There are well-identified energy sources for the inertial and baroclinic tidal peaks: fluctuations in the atmospheric windstress and the barotropic tide interacting with topography. The energy source for the continuum has not been identified yet. It has been conjectured that energy is scattered out of the inertial and baroclinic tidal peaks into the continuum, although detailed mechanisms and quantitative estimates are lacking. Other possible sources are surface waves, bottom flow over rough topography, mixed layer turbulence, and continuous geostrophic adjustment of large-scale flows.

Internal waves support diapycnal mixing in the ocean interior, at a rate equivalent to a diapycnal diffusivity of $10^{-7} \mathrm{~m}^{2-1} \mathrm{~s}^{-1}$. This is consistent with estimates inferred from microstructure measurements but insufficient to maintain the abyssal stratification which requires a value of $10^{-4} \mathrm{~m}^{2} \mathrm{~s}$, one order of magnitude larger. Microstructure measurements near topography indicate that boundary mixing may account for the required value in deep abyssal basins with their abundant topography but probably not in the pycnocline, unless observations have missed mixing "hot spots." Internal waves reflecting off and scattering at topography can provide the mechanical energy source for boundary mixing, but the exact mechanisms and their exact contribution have not been reliably determined yet.

Most of the energy sources in Figure 5 are external or boundary sources. Wind, surface waves, mixed layer turbulence, and barotropic tides and currents interacting with topography all generate internal waves at the boundaries of the ocean. The waves then propagate into the ocean interior. Only the generation due to continuous geostrophic adjustment is an interior source. This has important consequences for the parameterization of internal wave induced fluxes and internal wave induced mixing in oceanic general circulation models. If continuous geostrophic adjustment is the primary ener- gy source, then perhaps classical cascade arguments apply and the effect of internal waves might be parameterized solely in terms of local mean flow quantities. If boundary sources are dominant, this cannot be expected. Instead, the fluxes and flux divergences need to be calculated from the solution of a radiative transport equation for the internal wave field. Internal waves then represent an additional radiative field in oceanic general circulation models in the same way that light from the sun is treated as an additional radiative field in atmospheric general circulation models. To do such a radiative transport calculation requires that we know the energy pathways through the internal wave field. To understand these pathways is the primary focus of current internal wave research.

\section{Acknowledgments.}

We thank the participants of the workshop for their input into this report and for their permission to quote unpublished material. Copies of the proceedings are available from Peter Müller, University of Hawaii, School of Ocean and Earth Science, and Technology, Department of Oceanography, 1000 Pope Road, Honolulu, Hawaii, 96822. The eleventh 'Aha Huliko'a Hawaiian Winter Workshop was supported by the Department of the Navy grant number N00014-98-10207, issued by the Office of Naval Research.

\section{REFERENCES}

Briscoe, M.G., 1983: Observations on the energy balance of internal waves during Jasin. Phil. Trans. R. Soc. London, A308, 427-444.

Garrett, C.J.R. and W.H. Munk, 1972: Space-time scales of internal waves. Geophys. Fluid Dynn, 2, 225-264.

Müller, P., D. Olbers and J. Willebrand, 1978: The IWEX spectrum. J. Geophlys. Res., 83, 479-500.

Munk, W. and C. Wunsch, 1997: The moon, of course. Oceanography, 10, 132-134.

Munk, W. and C. Wunsch, 1998: Abyssal recipes II: energetics of tidal and wind mixing. Deep-Sea Res., 45, 1997-2010.

Wunsch, C., 1976: Geographical variability of the internal wave field: A search for sources and sinks. J. Phlys. Oceanogr., 6, 471-485. 